\title{
The feasibility of ultrasound Graf method in screening infants and young children with congenital hip dysplasia and follow-up of treatment effect
}

\author{
Dandan Liu ${ }^{1 \#}$, Xiaping Mou ${ }^{2 \#}$, Gang Yu ${ }^{3}$, Weixiang Liang ${ }^{1}$, Chun Cai ${ }^{3}$, Xiao Li $^{3}$, Gang Zhang ${ }^{3}$ \\ ${ }^{1}$ Department of Ultrasound Medicine, the Third Affiliated Hospital of Guangzhou Medical University, Guangzhou, China; ${ }^{2}$ Department of \\ Orthopedics, the People's Hospital of Jianyang City, Jianyang, China; ${ }^{3}$ Department of Pediatric Surgery, the Third Affiliated Hospital of Guangzhou \\ Medical University, Guangzhou, China \\ Contributions: (I) Conception and design: D Liu, X Mou, G Zhang; (II) Administrative support: W Liang; (III) Provision of study materials or \\ patients: D Liu, X Mou, G Yu, C Cai, X Li; (IV) Collection and assembly of data: All authors; (V) Data analysis and interpretation: W Liang, C Cai, \\ X Li, G Zhang; (VI) Manuscript writing: All authors; (VII) Final approval of manuscript: All authors. \\ \#These authors contributed equally to this work as co-first authors. \\ Correspondence to: Gang Zhang. Department of Pediatric Surgery, the Third Affiliated Hospital of Guangzhou Medical University, 63 Duobao Road, \\ Liwan District, Guangzhou 510150, China. Email: utopia21cn@126.com.
}

Background: Congenital hip dysplasia is a common limb deformity in infants and young children. This study aimed to clarify the feasibility of ultrasound Graf method in screening congenital hip dysplasia of infants and young children, and its application value in follow-up treatment.

Methods: A total of 1,313 infants and young children with clinically suspected congenital hip dysplasia in our hospital from December 2016 to January 2018 were selected as the participants and were examined by ultrasound Graf method. The acetabulum shape and the measured values of $\alpha$ and $\beta$ angles of the participants were observed. The development of the hip joint and distribution of congenital hip dysplasia were analyzed, and the treatment effect was followed up.

Results: Among 1,313 infants and young children with suspected congenital hip dysplasia, the positive rate of congenital hip dysplasia was $6.02 \%(79 / 1,313)$. The lesions were located on both sides in 14 cases, on the left side in 67 cases, and on the right side in 26 cases. The $\alpha$ angle of cases with congenital hip dysplasia was significantly lower than that of normal cases, and the $\beta$ angle was significantly higher than that of normal cases $(\mathrm{P}<0.05)$. Ultrasound follow-up results showed that out of 24 cases who underwent hip abduction exercises, 22 (91.67\%) returned to normal, and the remaining 2 returned to normal after Pavlik sling treatment. Among 46 cases treated with Pavlik sling, 42 (91.30\%) returned to normal, and the remaining 4 cases returned to normal after closed reduction and plaster fixation. A total of 9 participants underwent plaster fixation after closed reduction, all of which returned to normal.

Conclusions: Ultrasound Graf method can be used as the first choice for screening infants and young children with congenital hip dysplasia. It can be followed up to observe the clinical treatment effect, and it has high clinical application value.

Keywords: Ultrasound Graf method; congenital hip dysplasia; infants and young children; follow-up

Submitted Mar 04, 2021. Accepted for publication Apr 29, 2021.

doi: $10.21037 / \mathrm{tp}-21-137$

View this article at: http://dx.doi.org/10.21037/tp-21-137 


\section{Introduction}

Congenital hip dysplasia is one of the major clinical congenital malformations, and it has a high disability rate for infants and young children (1). Early screening and diagnosis after birth, and performing effective treatment in a timely manner is key to reducing the disability rate and improving the prognosis of infants and young children (2). Imaging methods are currently the main clinical auxiliary methods for infants and young children with congenital hip dysplasia, including X-ray, ultrasound, and others. However, $\mathrm{X}$-rays are limited in infant disease screening due to insufficient resolution and radiation (3). Ultrasonography has been widely used and popularized in the early screening of infant diseases due to its advantages including easy operation, repeatability, and no radiation damage (4). Based on this, this study analyzed the value of ultrasound Graf method in the screening and follow-up of treatment effects in infants and young children with congenital hip dysplasia, so as to provide a reliable basis for clinical diagnosis and treatment. We present the following article in accordance with the STROBE reporting checklist (available at http:// dx.doi.org/10.21037/tp-21-137).

\section{Methods}

\section{General information}

A total of 1,313 infants and young children with clinically suspected congenital hip dysplasia in our hospital from December 2016 to January 2018 were selected as the research participants. There were 660 males and 653 females, aged 28 days to 6 months, with an average of $3.25 \pm 0.59$ months. The inclusion criteria were as follows: (I) aged $\leq 8$ months; (II) patients with abnormal hip joint manifestations such as unequal length of lower limbs, limited hip joint abduction, and asymmetry of thigh skin patterns; (III) patients with complete clinical data; (IV) the family members of the participants were informed and signed an informed consent form. The exclusion criteria were as follows: (I) patients with multiple joint spasm and cerebral palsy; (II) patients with heart, liver, and kidney dysfunction; (III) patients with hematopoietic and immune system diseases; (IV) patients with other malignant tumors; (V) patients with hip joint dislocation caused by suppurative arthritis of the hip. This study was approved by the Third Affiliated Hospital of Guangzhou Medical University (2020-134). The study was conducted were in accordance with the Declaration of Helsinki (as revised in 2013). And informed consent was taken from all individual participants.

\section{Detection method}

The ultrasound Graf method was performed with the inspection instrument of GE ultrasonic diagnostic equipment (GE, Chicago, IL, USA), using a linear array probe of 9.0-15.0 MHz. The participants were checked while sleeping or in a quiet state, in the lateral position, with the lower limbs naturally bent and slightly internally rotated, and supported by a parent or assistant. The ultrasound probe was placed on the outer side of the greater trochanter of the femur, parallel to the long axis of the body, then the coronal surface of the femoral head and acetabulum were scanned, and a multi-section scan was performed to show the ischium of the inner femoral head. After acquiring the standard coronal image of the hip joint, the images were locked. The standard 7 images included the osteochondral interface, synovial reentry point, labrum, iliac plane, ilium turning point, ilium lowest point, and bony acetabular roof. The images were measured with the measuring tool of the instrument according to the following steps. The first step was to draw three measurement lines: the baseline (parallel or tangent to the iliac body), bony acetabular line (taking the lowest point of the iliac bone as the axis, and tangent to the bony acetabulum) and cartilaginous acetabulum line (the line between the turning point of the iliac bone and the midpoint of the labrum). The angle between the baseline and the bony acetabular line was defined as $\alpha$ angle, and the angle between the baseline and the cartilaginous acetabular line was defined as $\beta$ angle. The diagnostic criteria (5) are shown in Table 1.

\section{Observation indicators}

The results of ultrasound detection were analyzed by two or more experienced professional physicians to observe the shape of the participants' acetabulum and the measurement values of $\alpha$ and $\beta$ angles. The measurement results were averaged as the final result. Then, the ultrasound Graf method was used to analyze the development of the participants' hips and the distribution of congenital hip dysplasia, as well as follow up the treatment effect.

\section{Statistical methods}

In this study, data were analyzed using SPSS 22.0 software (IBM Corp., Armonk, NY, USA). The measurement data 
Table 1 Diagnostic criteria of the classification of Graf methods

\begin{tabular}{llll}
\hline Graf methods & $\begin{array}{l}\text { Parietal bone/ } \alpha \text { angle of the parietal } \\
\text { bone line }\end{array}$ & Parietal bone edge & Cartilage apex/ $\beta$ angle of the cartilage apex line \\
\hline Type I & $\begin{array}{l}\text { Good shape, head and acetabular in } \\
\text { counter position } \alpha \geq 60^{\circ}\end{array}$ & Rounded or sharp & Good femoral head coverage, la: $\beta<55^{\circ} ; \mathrm{lb}: \beta>55^{\circ}$ \\
Type II & $\begin{array}{l}\text { A bit poor of shape, no dislocation, } \alpha \\
\left(43^{\circ}-60^{\circ}\right) ; \text { Ila } / \mathrm{b}: 50^{\circ}-60^{\circ} ; \text { Ilc: } 43^{\circ}-50^{\circ}\end{array}$ & Rounded or curved & Femoral head coverage, $\beta=55^{\circ}-77^{\circ}$ \\
Type III & $\begin{array}{l}\text { Poorly shaped, dislocation of femoral } \\
\text { head, } \alpha<43^{\circ}\end{array}$ & Flat or stepped & $\begin{array}{l}\text { The femoral head is pressed upward and the } \\
\text { proximal perichondrium is pressed upward and } \\
\text { close to the iliac wall, } \beta \geq 77^{\circ}\end{array}$ \\
& $\begin{array}{l}\text { Poorly shaped, dislocation of femoral } \\
\text { head, } \alpha<43^{\circ}\end{array}$ & Flat or stepped & $\begin{array}{l}\text { The femoral head is pressed downward and the } \\
\text { proximal perichondrium is embedded between the } \\
\text { femoral head and the iliac wall }\end{array}$ \\
\hline
\end{tabular}

were described by the mean \pm standard deviation $(\bar{x} \pm s)$, and analyzed using $t$-test. The difference was statistically significant when $\alpha=0.05$.

\section{Results}

\section{Results of ultrasound Graf screening}

Among the 1,313 suspected cases with congenital hip dysplasia, there were 1,234 cases with normal hips (type I, see Figure 1A), accounting for $93.98 \%$; 31 cases of immature hip joint hip (type IIa, see Figure 1B), accounting for $2.36 \%$; and 48 cases of abnormal hip joints (type IIbIV, see Figure 1C,D,E), accounting for $3.73 \%$. The positive rate of congenital hip dysplasia examination was $6.02 \%$ (79/1,313), including 14 cases with bilateral congenital hip dysplasia, 67 cases with congenital hip dysplasia on the left side, and 26 cases with congenital hip dysplasia on the right side (Table 2).

\section{Comparison of $\alpha$ and $\beta$ angles of normal bip joint and congenital hip dysplasia by ultrasound Graf method}

The $\alpha$ angle of cases with congenital hip dysplasia was significantly lower than that of normal cases, and the $\beta$ angle was significantly higher than that of normal cases. The differences between the groups were statistically significant $(\mathrm{P}<0.05$, Table 3).

\section{Treatment of 79 cases}

Among 79 cases with congenital hip dysplasia, 24 participants (30.38\%) were treated by hip abduction exercises; 46
(58.23\%) were treated by Pavlik sling; and 9 (11.39\%) were treated by closed reduction and plaster fixation under general anesthesia (Table 4).

\section{Follow-up results of ultrasound Graf method}

Participants undergoing hip abduction exercises and Pavlik sling treatment underwent color Doppler ultrasound review every 2-3 weeks until they returned to normal or had been treated more than half a year; cases undergoing closed reduction and plaster fixation underwent color Doppler ultrasound review every 3-6 months for a duration of 4 months to 3 years. The deadline for follow-up was January 2021. All participants completed follow-up with a follow-up rate of $100 \%$. Among 24 the participants with hip abduction exercises, 22 (91.67\%) returned to normal, and the remaining 2 participants returned to normal after Pavlik sling treatment. Among the 46 participants who underwent Pavlik sling treatment, 42 (91.30\%) returned to normal afterwards, and the remaining 4 participants returned to normal after closed reduction and plaster fixation. And there were 9 cases of plaster fixation after closed reduction, and all of them returned to normal.

\section{Analysis of ultrasonic images}

The ultrasound images of different types of hip joint were different and shown in Figure 1.

\section{Discussion}

Congenital hip dysplasia is a common limb deformity in 

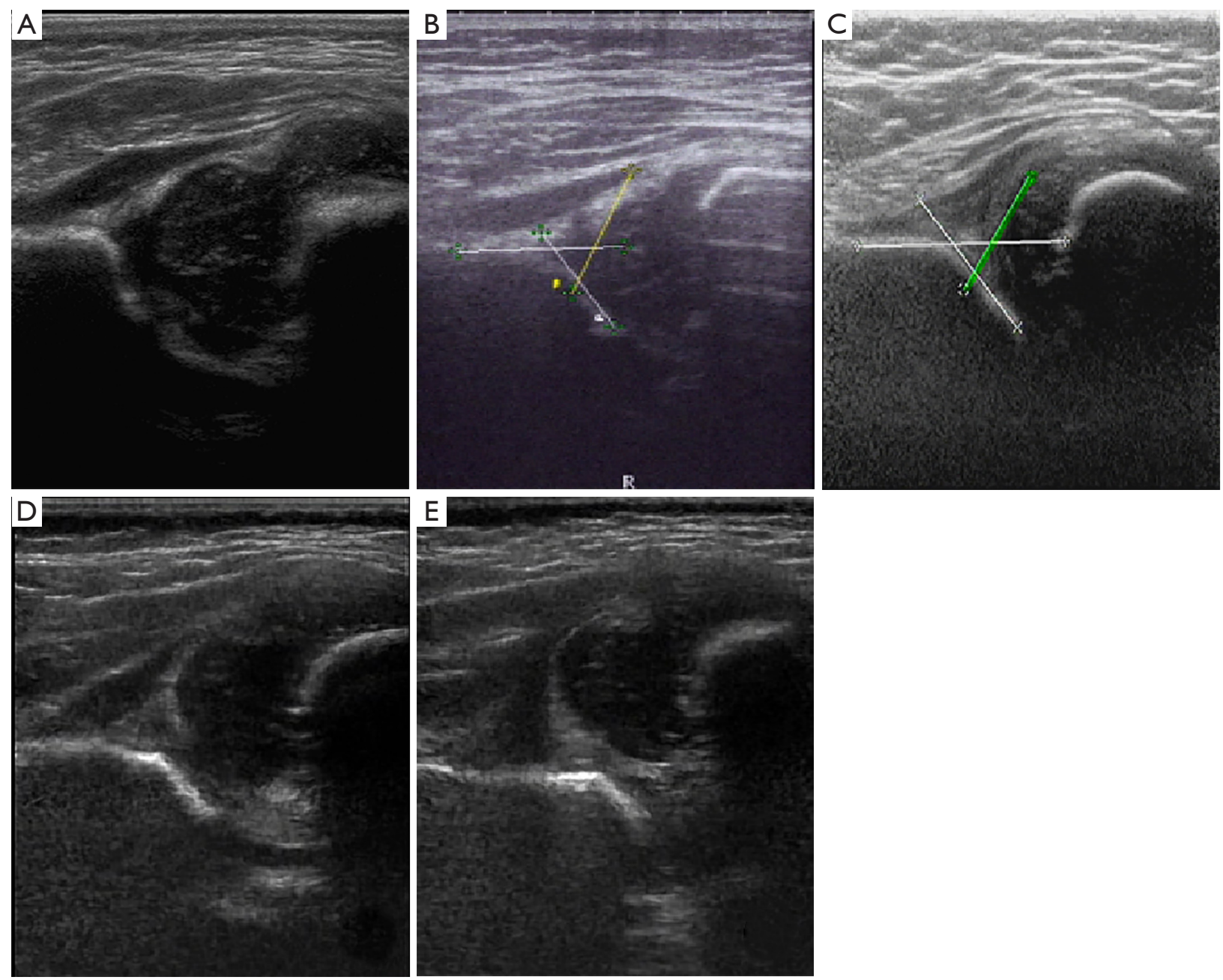

Figure 1 Ultrasonic images of different hip joints. (A) Type I hip joint. The ultrasound image showed that the acetabulum was in good shape and the parietal bone edge was slightly rounded and blunt; (B) Type IIa/b hip joint. The ultrasound image showed that the acetabulum had a slightly poor shape without dislocation, and the parietal bone edge was curved; (C) Type IIc hip joint. The ultrasound image showed that the acetabulum was poorly shaped, not dislocated, and the parietal bone edge was flat; (D) Type III hip joint. The ultrasound image showed that the acetabulum was poorly shaped, dislocated, and the femoral head was compressed upward; (E) Type IV hip joint. The ultrasound image showed that the acetabulum was poorly shaped, dislocated, the femoral head was pressed downward, and the proximal perichondrium was embedded between the femoral head and the ilium wall.

infants and young children, ranking first in limb deformity diseases, with an incidence of about $0.15-0.20 \%$ (6). This study screened infants and young children with positive clinical signs, and the results showed that the positive rate of ultrasound examination was $6.02 \%$, slightly higher than that in the previous study (7). The possible reason of the increase may be related to the maturity of sonographers' operating technology, development of equipment, and spread of diseases in recent years. In addition to ultrasound, clinical physical examination, $\mathrm{X}$-ray film, and magnetic resonance imaging (MRI) are the other main methods for clinical diagnosis of hip joint diseases in infants and young children (8). Although low-dose CT reduces the radiation dose, the image quality of infants is easy to be poor due to the age problem, and the radiation dose still exists. Although MRI has high accuracy, the scanning time is too long and the price is too expensive, so it is not suitable for preliminary diagnosis. However, compared with plain radiographs and MRI, hip ultrasound examination has the advantages of being effective, non-invasive, and low-cost, 
Table 2 Results of ultrasound Graf screening

\begin{tabular}{|c|c|c|c|c|c|c|c|}
\hline \multirow{2}{*}{ Classification of hip joint } & \multicolumn{2}{|c|}{ Gender (cases) } & \multicolumn{3}{|c|}{ Sites (cases) } & \multirow{2}{*}{ Total (cases) } & \multirow{2}{*}{ Detection rate (\%) } \\
\hline & Male & Female & Left side & Right side & Bilateral & & \\
\hline I & 618 & 616 & - & - & - & 1,234 & 93.98 \\
\hline Ila & 16 & 15 & 29 & 9 & 7 & 31 & 2.36 \\
\hline Ilb & 7 & 7 & 11 & 6 & 3 & 14 & 1.07 \\
\hline Ilc & 7 & 6 & 11 & 4 & 2 & 13 & 0.10 \\
\hline III & 8 & 6 & 11 & 4 & 1 & 14 & 1.07 \\
\hline IV & 4 & 3 & 5 & 3 & 1 & 7 & 0.05 \\
\hline
\end{tabular}

Table 3 Comparison of $\alpha$ and $\beta$ angles of normal hip joint and congenital hip dysplasia by ultrasound Graf method $\left(\bar{x}_{ \pm}\right)$

\begin{tabular}{lcccc}
\hline & Normal hip joint $(\mathrm{n}=1,234)$ & Congenital hip dysplasia $(\mathrm{n}=79)$ & $\mathrm{t}$ & $\mathrm{P}$ value \\
\hline A angle $\left(^{\circ}\right)$ & $64.13 \pm 4.63$ & $37.02 \pm 3.62$ & 51.047 & $<0.001$ \\
B angle $\left(^{\circ}\right)$ & $40.47 \pm 3.51$ & $111.62 \pm 19.72$ & 104.04 & $<0.001$ \\
\hline
\end{tabular}

Table 4 Treatment of 79 children [n (\%)]

\begin{tabular}{lccc}
\hline Classification of hip joint & Hip abduction exercises & Pavlik sling & Closed reduction and plaster fixation \\
\hline IIa $(n=31)$ & $20(64.52)$ & $11(35.48)$ & $0(0.00)$ \\
IIb $(n=14)$ & $4(28.57)$ & $10(71.43)$ & $0(0.00)$ \\
IIC $(n=13)$ & $0(0.00)$ & $9(69.23)$ & $4(30.77)$ \\
III $(n=14)$ & $0(0.00)$ & $11(78.57)$ & $3(21.43)$ \\
IV $(n=7)$ & $0(0.00)$ & $5(71.43)$ & $2(28.57)$ \\
Total & $24(30.38)$ & $46(58.23)$ & $9(11.39)$ \\
\hline
\end{tabular}

especially for infants whose hip joints are mainly cartilage, therefore the results of hip ultrasound examination are more reliable (9). Ultrasound Graf method can be used to evaluate development of the hip joint by analyzing the ultrasound images of the hip joint and quantifying the shape of the acetabulum with $\alpha$ and $\beta$ angles (10). The results of this study showed that there was a significant difference in $\alpha$ and $\beta$ angles between the cases with congenital acetabular dysplasia and those with normal hip joints by ultrasound Graf method, which is consistent with the reports of Fooladi (11), suggesting that the ultrasound Graf method can be used as one of the methods to screen congenital acetabular abnormalities, with strong operability and high standardization.

Related international reports have shown that the incidence of children with congenital hip dysplasia differs according to race, region, and gender. Compared with those of eastern and southeast Asian heritage, the incidence among Caucasians is lower: the incidence in China is about $0.1-0.6 \%$, and the incidence in the United States is about $0.1 \%$. Besides, the incidence of congenital hip dysplasia is higher on the left side than on the right $(12,13)$. Among participants with congenital hip dysplasia in this study, the left side was the most commonly affected, with an incidence of $6.02 \%$, which was basically consistent with the reports of the above-mentioned literatures. If patients with abnormal hip joints are not treated in time, they may develop severe osteoarthritis, claudication, or other serious joint dysfunction, which can even cause lifelong deformities. It is believed that timely diagnosis and treatment are the keys to improving the prognosis of patients with congenital hip dysplasia (14).

Most immature hip cases with an $\alpha$ angle of $55^{\circ}-60^{\circ}$ 
can spontaneously develop to maturity during follow-up. Therefore, the guardians of such children can be educated to perform straddling hugs or hip abduction exercises, and eventually most of these children will return to normal during the course of development $(15,16)$. In the followup study, $91.67 \%$ of the 24 participants treated with hip abduction exercises returned to normal, indicating that the treatment effect is good. Patients treated with this method need to be re-examined after $2-3$ weeks. If an ultrasound result shows that there is no significant change in the $\alpha$ angle or the clinical symptoms have not been improved, they a Pavlik sling or other treatments should be applied. Pavlik sling therapy is suitable for all types of patients with congenital hip dysplasia within 6 months (17). Patients treated with Pavlik sling therapy should undergo regular ultrasound follow-up once every 2 to 3 weeks. If the ultrasound result reveals a prompt reset after 3 weeks, the treatment should be continued for 2 to 4 months. If ultrasound and clinical examination shows no reset after 3 weeks, Pavlik sling treatment should be discontinued and other therapies applied (18). In this study, among the 46 children who underwent Pavlik sling treatment, ultrasound follow-up results showed that the effective rate of treatment was $91.30 \%$. Plaster fixation after closed reduction and open reduction therapies are suitable for cases who have failed to be cured after the above methods. In the clinical selection of treatment therapies, comprehensive evaluation should be performed based on the age of the patient, and development of the femoral head and acetabulum. The role of ultrasound examination has shown to be important in evaluating the development of the femoral head and acetabulum (19). In addition, ultrasound examination has a good evaluation value in the treatment of congenital hip dysplasia, and can effectively guide the choice of clinical treatment options. However, with the deepening of ultrasound screening, there are still some problems: Firstly, it is still controversial whether ultrasound screening should be universal or only performed for cases with positive clinical signs of hip dysplasia (20); Secondly, the shape of the acetabulum and the measured alpha and beta angles are subject to some subjective factors, and the results may be biased; Thirdly, as mild congenital dysplasia of the hip involves the phenomenon of spontaneous development and maturity, especially for children with an $\alpha$ angle of $55^{\circ}-60^{\circ}$, the risk of ultrasound overuse for diagnosis and subsequent waste of medical resources and whether there are false negative clinical signs and the value of ultrasound in the early diagnosis of false negative clinical signs of hip dysplasia remain to be further assessed.

In summary, the ultrasound Graf method can be used as the first choice for screening infants and young children with congenital hip dysplasia, it can also be used for followup observation of clinical treatment effects, and has high application value in clinic.

\section{Acknowledgments}

Funding: None.

\section{Footnote}

Reporting Checklist: The authors have completed the STROBE reporting checklist. Available at http://dx.doi. org/10.21037/tp-21-137

Data Sharing Statement: Available at http://dx.doi. org/10.21037/tp-21-137

Conflicts of Interest: All authors have completed the ICMJE uniform disclosure form (available at http://dx.doi. org/10.21037/tp-21-137). The authors have no conflicts of interest to declare.

Ethical Statement: The authors are accountable for all aspects of the work in ensuring that questions related to the accuracy or integrity of any part of the work are appropriately investigated and resolved. This study was approved by the Third Affiliated Hospital of Guangzhou Medical University (2020-134). The study was conducted were in accordance with the Declaration of Helsinki (as revised in 2013). And informed consent was taken from all individual participants.

Open Access Statement: This is an Open Access article distributed in accordance with the Creative Commons Attribution-NonCommercial-NoDerivs 4.0 International License (CC BY-NC-ND 4.0), which permits the noncommercial replication and distribution of the article with the strict proviso that no changes or edits are made and the original work is properly cited (including links to both the formal publication through the relevant DOI and the license). See: https://creativecommons.org/licenses/by-nc-nd/4.0/.

\section{References}

1. Lange AE, Lange J, Ittermann T, et al. Population-based 
study of the incidence of congenital hip dysplasia in preterm infants from the Survey of Neonates in Pomerania (SNiP). BMC Pediatr 2017;17:78.

2. Shorter D, Hong T, Osborn DA. Screening programmes for developmental dysplasia of the hip in newborn infants. Cochrane Database Syst Rev 2011;2011:CD004595.

3. Hegde D, Powers N, Nathan EA, et al. Developmental dysplasia of the hip in preterm breech infants. Arch Dis Child Fetal Neonatal Ed 2020;105:556-8.

4. Aarvold A, Schaeffer EK, Kelley S, et al. Management of Irreducible Hip Dislocations in Infants With Developmental Dysplasia of the Hip Diagnosed Below 6 Months of Age. J Pediatr Orthop 2019;39:e39-e43.

5. Walton S, Schaeffer E, Mulpuri K, et al. Evaluating the role of prereduction hip traction in the management of infants and children with developmental dysplasia of the hip (DDH): protocol for a systematic review and planned meta-analysis. BMJ Open 2018;8:e019599.

6. Atalar H, Gunay C, Komurcu M. Functional treatment of developmental hip dysplasia with the Tübingen hip flexion splint. Hip Int 2014;24:295-301.

7. Jawadi AH, Wakeel A, Tamimi W, et al. Association analysis between four vitamin $\mathrm{D}$ receptor gene polymorphisms and developmental dysplasia of the hip. J Genet 2018;97:925-30.

8. Willemsen K, Doelman CJ, Sam ASY, et al. Long-term outcomes of the hip shelf arthroplasty in adolescents and adults with residual hip dysplasia: a systematic review. Acta Orthop 2020;91:383-9.

9. Labianca L, Gajaseni P, Dolan L, et al. Pemberton Pericapsular Osteotomy for Developmental Dysplasia of the Hip and Hip Dislocation - Honorable Mention. J Am Acad Orthop Surg 2018;26:175.

10. Abounahia FF, Abu-Jarir R, Abounahia MF, et al. Prophylactic Sildenafil in Preterm Infants at Risk of Bronchopulmonary Dysplasia: A Pilot Randomized, Double-Blinded, Placebo-Controlled Trial. Clin Drug Investig 2019;39:1093-107.

11. Fooladi S, Kundu T. Ultrasonic field modeling in anisotropic materials by distributed point source method.

Cite this article as: Liu D, Mou X, Yu G, Liang W, Cai C, Li X, Zhang G. The feasibility of ultrasound Graf method in screening infants and young children with congenital hip dysplasia and follow-up of treatment effect. Transl Pediatr 2021;10(5):1333-1339. doi: 10.21037/tp-21-137
Ultrasonics 2017;78:115-24.

12. Rosenkrantz E, Bottero A, Komatitsch D, et al. A flexible numerical approach for non-destructive ultrasonic testing based on a time-domain spectral-element method: Ultrasonic modeling of Lamb waves in immersed defective structures and of bulk waves in damaged anisotropic materials. NDT \& E International 2019;101:72-86.

13. Graf M, Riedel T. Electrical impedance tomography: Amplitudes of cardiac related impedance changes in the lung are highly position dependent. PLoS One 2017;12:e0188313.

14. Beraich M, Shaili H, Benhsina E, et al. Preparation and characterization of $\mathrm{Cu} 2 \mathrm{FeGeS} 4$ thin-film synthesized via spray ultrasonic method DFT study. Materials Letters 2020;275:128070.

15. Siddiqui N, Aeri V. Ultrasonic extraction optimization of stigmasterol using response surface methodology and quantification by high-performance thin-layer chromatography from Tecomella undulata bark. JPC - Journal of Planar Chromatography - Modern TLC 2017;30:36-42.

16. Mehmood T, Ahmad A, Ahmed A, et al. Optimization of olive oil based $\mathrm{O} / \mathrm{W}$ nanoemulsions prepared through ultrasonic homogenization: A response surface methodology approach. Food Chem 2017;229:790-796.

17. Mirza H, Garcia J, McKinley G, et al. Duration of significant patent ductus arteriosus and bronchopulmonary dysplasia in extremely preterm infants. J Perinatol 2019;39:1648-55.

18. Bercik D, Diemer S, Westrick S, et al. Relationship between torticollis and gastroesophageal reflux disorder in infants. Pediatr Phys Ther 2019;31:142-7.

19. Dibello D, Odoni L, Pederiva F, et al. MRI in Postreduction Evaluation of Developmental Dysplasia of the Hip: Our Experience. J Pediatr Orthop 2019;39:449-52.

20. Alassaf N. Prediction of the requirement of open reduction for developmental dysplasia of the hip. J Int Med Res 2018;46:54-61.

(English Language Editor: J. Jones) 